\title{
New United States mammogram guidelines ignite debate
}

Published at www.cmaj.ca on Dec. 5, 2009

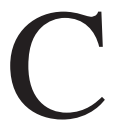
hanges to mammogram guidelines in the United States have earned praise from some cancer advocacy groups, attracted scorn from others and appear to have confused much of the public.

On Nov. 16, 2009, the United States Preventative Services Task Force, a 16member panel of experts appointed by the Department of Health and Human Services, released new guidelines for breast cancer screening. The guidelines recommend that women aged 50-74 have mammograms every two years instead of annually. The most controversial update, however, is the new guideline suggesting that regular breast cancer screening begin at age 50 instead of 40, as was suggested in the old guidelines.

Some critics of the new guidelines say the task force was politically motivated, making changes only to save money as part of US President Barack Obama's health care reform plan. Others opposed to the changes say they will lead to private health insurers restricting screening reimbursement, as well as Medicaid and Medicare cutting back on coverage for mammograms, which will hurt low-income women.

"I know that some have argued that for the underserved women, screening is their access to the health care system. But that is why we are working so hard to have laws that guarantee access to care for all," Fran Visco, president of the Washington, DC-based National Breast Cancer Coalition, wrote in an email. "The underserved are entitled to the truth just as much as those that are comfortable accessing the health care system. We should put our efforts toward outreach to educate that population about quality and evidence and access, rather than continue to send messages that are incorrect."

For women aged 50-59, one death is prevented for every 1339 women screened for 10 years, the task force concluded. But among women aged

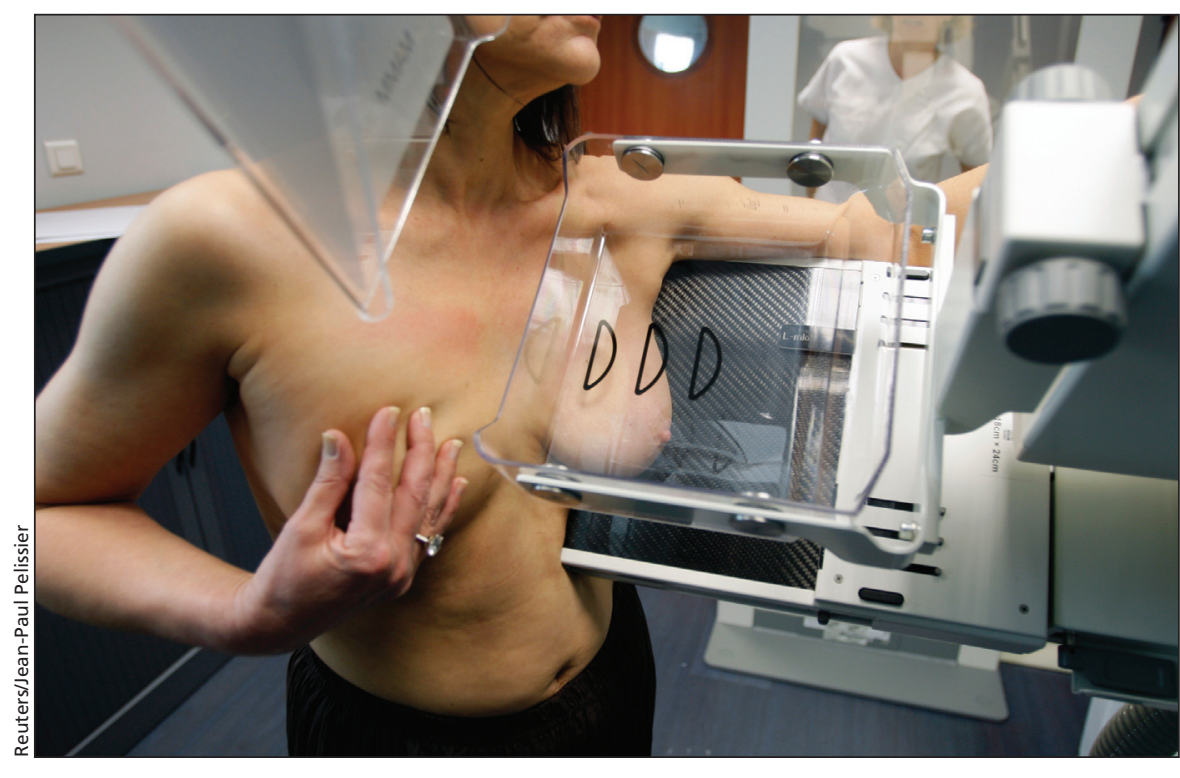

Experts in the United States are questioning the efficacy of mammograms for women under 50 , saying the risks of incorrect diagnosis outweigh the benefits of early screening.

40-49, one death is prevented for every 1904 screened. The task force concluded that this risk is not enough to recommend widespread screening for women in their 40s considering the counter risk of incorrect diagnoses.

"The benefits of implementing the recommendations of the Task Force for women in their 40 s include fewer unnecessary biopsies, less overtreatment and the resulting unnecessary toxic and life threatening treatments, cost savings that can be redirected to finding answers that are correct for women in this age group and better understanding by the public about the complexity of breast cancer and the importance of evidence based medical care," Visco wrote.

Though positively received by some cancer support groups, others were quick to denounce the new guidelines. The American Cancer Society, for example, announced it would continue to recommend screening for women in their 40s and claimed the task force did not consider all available evidence. In a press release, Dr. Otis Brawley, the society's chief medical officer, wrote that "the overall effectiveness of mammography increases with increasing age. But the limitations do not change the fact that breast cancer screening using mammography starting at age 40 saves lives. As someone who has long been a critic of those overstating the benefits of screening, I use these words advisedly: this is one screening test I recommend unequivocally, and would recommend to any woman 40 and over, be she a patient, a stranger, or a family member."

Though the move to target only women at least 50 years of age has stirred controversy in the US, this is, for the most part, standard practice in Canada. In Canada, each province conducts its own breast cancer screening program. Some provinces, such as Quebec and Nova Scotia, suggest women 50-plus years of age have mammograms every two years. Other provinces, such as Ontario and British Columbia, recommend annual tests. In all provinces, women aged 50 and above have unfettered access to these programs. In others, however, women in their 40s must obtain physician referrals to have mammograms. 
Canada is not directly affected by the new US guidelines, but some Canadian cancer advocacy groups say they may cause people to question the value of mammography. "They've increased confusion about who should participate in breast cancer screening and what the benefits are," says Beth Easton, director of allocations and health promotions for the Canadian Breast Cancer Foundation. "All the [Canadian Breast Cancer] Foundation wishes to see is a fair interpretation of the evidence and a fair presentation of the risk. The guidelines and the media discussion will leave women with the impression that mammograms cause harm and won't provide a clear understanding of the significant benefits that mammograms can have for women in a range of age groups, including women aged 40 to 49 ."
The confusion surrounding the new guidelines, some critics claim, will cause people to lose confidence in the medical profession. Gail Collins, a breast cancer survivor who writes for The New York Times, expressed this opinion in a recent column. Collins had received mammograms regularly, she wrote, and yet her cancer, which her oncologist suggested developed because of estrogen replacement therapy, went undetected until she discovered a lump in her breast during a selfexam. "So, in summary," Collins wrote in a Nov. 19, 2009, column, "the cutting-edge of medical thinking of the 1990s may have induced my cancer, and then the universally recommended testing protocol failed to detect it."

Though it will inevitably confuse some people, periodically updating breast cancer screening recommendations is necessary, says Heather Chappell, director of cancer control policy for the Canadian Cancer Society. "We always want to be refining guidelines based on the most recent evidence."

Chappell also notes that the United States Preventative Services Task Force did not say that women in their 40 s could not or should not have mammograms, but that women in this age group are in a greyer area than older women and should therefore make more deliberate decisions.

"They are recommending that those women have informed discussions with their doctors," says Chappell. "We have been encouraging that here for some time." - Roger Collier, CMAJ

DOI:10.1503/cmaj.109-3124 